\title{
ON THE LATENT STRUCTURE OF PROBLEM GAMBLING: A TAXOMETRIC ANALYSIS
}

Richard J. E. James, Claire O’Malley \& Richard J. Tunney

This is the peer reviewed version of the following article: James, R. J. E., O'Malley, C. \& Tunney, R. J. (2014). On the latent structure of problem gambling: A taxometric analysis, Addiction, 109(10), 1707-1717, which has been published in final form at http://dx.doi.org/10.1111/add.12648. This article may be used for non-commercial purposes in accordance with Willey Terms and Conditions for Self-Archiving (available at http://olabout.wiley.com/WileyCDA/Section/id-828039.html).

\section{Funding information}

This work was funded by the Economic and Social Research Council (ES/J500100/1) and the Engineering and Physical Sciences Research Council (EP/G037574/1). 


\begin{abstract}
Aims. To test whether problem gambling is a categorical or dimensional disorder on the basis of two problem gambling assessments. This distinction discriminates between two different conceptualizations of problem gambling, one that problem gambling is defined by its addictive properties, the other that it is a continuum of harm.

Setting. The British Gambling Prevalence Survey 2010, a nationally representative sample of the United Kingdom conducted by the National Centre for Social Research.

Method. Five different taxometric analyses were carried out on cases from two problem gambling screens; the Problem Gambling Severity Index and a measure derived from the DSM-IV Pathological Gambling criteria. Two analyses were conducted on the total scores for these measures.
\end{abstract}

Results. There was strong evidence that both scales were measuring a categorical construct. Fit indices consistently supported a categorical interpretation (CCFI's $>0.6$ ). The PGSI analysis indicated the presence of a taxon (CCFI's $=0.633,0.756)$. The analysis conducted on the adapted DSM-IV criteria indicated stronger quantitative support for a taxon $($ CCFI's $=0.717,0.811$ and 0.756$)$ but items probing a loss of control were inconsistent. The taxometric analyses of both scales support a categorical interpretation (CCFI's $=0.628$, 0.567), but should be extreme caution due to high nuisance covariance.

Conclusions. Both problem gambling screens analysed are likely to be measuring a categorical construct that taps into a categorical, loss of control model of problem gambling. There is some evidence that the two screens are measuring different aspects of an addiction construct. 


\section{Introduction}

A longstanding debate concerns the latent structure of problem gambling [1]. Two perspectives have emerged, one arguing that problem gambling is defined by a categorical division between gamblers and problem gamblers, typified by loss of control over gambling, the other that problem gambling is at the extreme of a continuum of harm and that problem gambling assessments form a useful but arbitrary cutting point. This debate is central to research on the theory and measurement of problem gambling and its relationship to other addictions. To address this question we conducted a taxometric analysis of two measures of problem gambling recorded in the British Gambling Prevalence Survey 2010 (BGPS 2010) [2]: The Problem Gambling Severity Index (PGSI; [3]) and a measure adapted from the DSM-IV Pathological Gambling criteria [2, 4].

There are two different conceptualizations of problem gambling [1]. The first defines problem gambling as an addiction disorder and a manifestation of pathological gambling. Pathological gambling is defined by this approach as a loss of control over gambling behaviour [5]. This category of theory includes models that emphasise causal roles for biological, psychological and social factors, in addition to loss of control in the development of problem gambling. For example the Pathways Model [6] proposes three distinct aetiologies of problem gambling. These pathways assume that problem gambling is caused by behavioural conditioning, life stressors, or impulsive/antisocial personality traits, the latter two underpinned by reinforcement learning and mounting cognitive biases, leading to a loss of control over gambling behaviour prior to the onset of pathological gambling. This model has strong empirical support, with several studies confirming three kinds of problem gambler [7, 8]. Although the Pathways Model is not purely an addiction-based theory, it does claim that a defining feature of problem gambling is a loss of control of gambling behaviour, that problem gambling is a categorical disorder and that different pathways are qualitatively 
distinct from one another. The model also claims that behaviourally conditioned gamblers, unlike the other two pathways, can return to controlled gambling. These claims imply the presence of a problem gambling taxon [5] or qualitatively distinct category.

An alternative approach to problem gambling focuses on the individual harm that problem gambling causes and the wider impact of gambling on others and society [9]. Although addictive gambling may be an important issue in this framework, this approach claims that the demarcation of problem gambling is excessive gambling behaviour [1]. Excessive gambling is defined as the continuation of gambling beyond the limits an individual's circumstances allow. In contrast to the Pathways Model, this conceptualization assumes that problem gambling is identified as a threshold along a dimension that includes non-problematic recreational gambling.

At first glance, research on the measurement of problem gambling reflects the same differences that exist in the theoretical literature. However, measures of problem gambling appear to exclusively measure an addiction construct $[1,10,11]$. The Problem Gambling Severity Index (PGSI) is the most common population-wide assessment of problem gambling [12]. The PGSI is designed to classify four levels of problem gambling severity along a dimension of harm [3]. Although the use of a measure that hypothesizes a dimensional structure to make a categorical distinction isn't problematic if that distinction is meaningful [13], the use of the PGSI has produced difficulties in the problem gambling prevalence literature as there has been a failure to find a consensus on the appropriate threshold to discriminate between recreational and problem gamblers and the validity of the PGSI categories [12, 14-19]. This debate arises because some items used in the PGSI to measure problem gambling as a dimensional construct are adapted from Pathological Gambling instruments such as the South Oaks Gambling Scale [20] and DSM-IV [1, 4]. 
Understanding whether problem gambling has a categorical or dimensional structure is particularly important with the inclusion of Gambling Disorder in the DSM-V as a behavioural addiction. This has been justified on the basis that behavioural, neural and genetic markers in pathological gamblers are similar to other addictions [21, 22]. However, one property that has not been tested is whether problem gambling has a similar latent structure to other addictions. Meta-analytic studies using taxometric analysis (a method to test whether the latent structure of a variable is categorical or dimensional) specifically identify addictions as one of the few psychopathologies that may be categorical [23]. Studies looking at the subtyping of problem gamblers have consistently found three subtypes of problem gambler [7, 8]. Despite considerable debate about the nature of problem gambling, few studies have been conducted using appropriate statistical methods to test whether problem gambling is categorical.

However, numerous studies using a variety of analytic methods including some taxometric analyses have also found evidence that addictive disorders are best described as dimensional. Some of these have used latent class and latent mixture methods, in addition to methods that presuppose the presence of a latent factor $[10,24]$. Some taxometric analyses looking at several substance abuse disorders [25, 26, 27] have found evidence that disputes the presence of a taxon [23]. Moreover a latent class analysis of British gamblers as part of the British Gambling Prevalence Survey 2007 indicated the presence of several latent classes ordered along symptom severity [28].

Two previous studies have conducted taxometric analyses on gambling. The first examined whether excessive internet sports gamblers formed a taxon [29]. Data were collected from actual internet gambling behaviour over several years. The analysis was carried out on three behavioural measures of involvement (money wagered, money lost and number of bets). Unfortunately for our purposes measures of problem gambling were not 
taken and the results from this study were inconclusive in discriminating between models. The second study examined whether problem gamblers formed a taxon on the basis of PGSI scores [19]. PGSI items were analysed from respondents who scored greater than zero in the South African National Urban Prevalence Study of Gambling Behaviour [30]. The taxometric analyses on these data indicated a categorical structure, and stronger support was found when the analysis was restricted to items testing a loss of control. However, the PGSI may not be the best measure to detect a problem gambling taxon because it contains fewer items relating to loss of control [19]. The DSM has a greater number of items relating to a loss of control but to date, no such analysis has been conducted.

To this end, and to determine whether problem gambling has a categorical or dimensional structure, we carried out taxometric analyses on the data from two problem gambling measures collected in the BGPS 2010 (PGSI and an adapted version of the DSMIV Pathological Gambling criteria) [2]. There are two benefits of using this dataset. First, as a general population dataset it contains the entire range of responses for both measures. Second, the DSM measure was collected in both continuous and dichotomous formats. Taxometric analyses typically require a variable to have at least 4 rank ordered categories in order to be suitable for analysis [31]. Although the logic of the DSM-IV assumes a categorical structure, previous analyses have found that the DSM-IV construct of Pathological Gambling may be dimensional $[10,11]$. This interpretation may be an artefact of the analytic techniques these studies have used (e.g. factor analysis, Rasch models). Taxometric analyses do not make this assumption and can discriminate between latent variables that have categorical or dimensional structures. We hypothesized that taxometric analyses of both the PGSI and DSM-IV would find evidence for a categorical structure potentially similar to other addictions. 


\section{Methods}

British Gambling Prevalence Survey 2010

The BGPS data collection consisted of a computer aided self-interview conducted by the National Centre for Social Research [2, 32]. Almost eight thousand $(N=7,756)$ respondents completed the survey (response rate $=47 \%$ ). Participants were randomly sampled from 391 postcodes, which were selected from a stratified probability sample. Data were analysed from participants who scored one or more on either the PGSI $(N=569)$ or the DSM $(N=1,387)$ measures, based on previous taxometric analyses of problem gambling screens [19]. Both measures assessed problem gambling prevalence over the past year. One case was removed prior to analysis as it did not contain a full set of responses. The BGPS estimated the 2009 problem gambling prevalence in the UK to be $0.9 \%$ (DSM-IV based measure) and $0.7 \%$ (PGSI). Distributional information about the data is included in Table 1.

Measures

The PGSI consists of nine questions that are scored from zero to three. Participants were classified as problem gamblers if they scored 8 or higher [2]. The PGSI has strong internal consistency ( $\alpha=0.9$ [11]). The classification accuracy of the PGSI has been studied alongside multiple measures and has been demonstrated to have adequate classification accuracy with the present problem gambling criteria (Positive Predictive Value $=89.86 \%$, Negative Predictive Value $=92 \%$, Sensitivity $=44.42 \%$, Specificity $=99.22 \%[33]$ )

The authors of the BGPS used a modified version of the DSM-IV Pathological Gambling criteria [2, 34-36]. Instead of scoring the presence or absence of a symptom the respondents rated each item on a four-point scale of the frequency each symptom occurred (0 being "never" and 3 "very often"). Also a lower threshold was used to classify problem 
gamblers (three) than the DSM-IV criteria (five). The authors of the BGPS justified this threshold [11] on the basis that a lower threshold is better at classifying these groups [37, 38].

The adapted DSM-IV criteria show adequate internal consistency $(\alpha=0.73$ [11], 0.76 [39]). $[2,34-36]$

Analytic Procedure

MAMBAC (Mean Above Minus Below A Cut; [40]) and MAXSLOPE (Maximum Slope; [41]) analyses were carried out on the PGSI indicator variables. MAMBAC, MAXCOV (Maximum Covariance; [42]) and MAXEIG (Maximum Eigenvalue; [43]) analyses were conducted on the adapted DSM-IV items.

Taxometric analyses require an input variable and output variables. Across all taxometric analyses cases are rank ordered by one of the variables selected for analysis (the input variable), which forms the $\mathrm{x}$-axis of taxometric plots (Figures 1-3). In MAMBAC analyses a series of cuts (preferably 50 [44]) are applied evenly across the other variable (the output variable). At each cut a mean difference, defined as the mean above minus the mean below is computed and plotted as the y-axis. The MAMBAC procedure is iterated through each potential input-output combination. MAXCOV analyses portions the input variable into a number of 'windows' or subsamples [31] as the input and the covariance between couplets of output variables at each window is plotted as the output variable. MAXEIG operates in a similar fashion to MAXCOV, except that the largest eigenvalue from two or more output variables is plotted. [31]. MAXSLOPE uses a slightly different approach, plotting a smoothed non-linear regression curve and is conducted on two indicator variables. Categorical taxometric plots are generally peaked, whereas dimensional plots are flat [13] 
For each analysis the observed data were compared against 200 samples of bootstrapped comparison data. Comparison data can discriminate between structures when the data are highly skewed [45]. The bootstrapped data had the same distributional statistics as the dataset, but half the samples had an idealised dimensional or a categorical structure. From this the root mean squared residual (RMSR) was computed as an index of fit between the bootstrapped and observed data, and an index of the latent structure was derived by dividing the RMSR for the dimensional data by the sum of the RMSR's for the categorical and dimensional data. This produced a comparison curve fit index (CCFI; [13]) between 0 and 1. Indices closer to 1indicate a categorical structure and smaller indices a dimensional structure. A CCFI of 0.5 indicates support for neither structure. CCFI's between 0.4 and 0.6 are inconclusive [13].

For both MAMBAC analyses, 50 evenly spaced cuts were made in the output variable, with the first and last cuts specified as the 10 cases reserved at either extreme, based on studies using bootstrapped data [44]. For MAXCOV and MAXEIG analyses, the output variables were divided into 50 windows.

All of the taxometric analyses was carried out using an $\mathrm{R}$ script developed by Ruscio [46].

\section{Results}

\section{Indicator Construction}

Cases were classified as problem or non-problem gambler based on each measure's classification criteria. A number of assumptions concerning the data should be met before taxometric analyses are conducted. The first recommends the base rate, or proportion of cases in the whole sample assigned to the putative taxon should be $>=10 \%$ [47] or $5 \%$ [13]. The PGSI base rate (0.086) is sufficient but the DSM-IV rate $(0.046)$ is smaller than the 
recommended heuristic. The second requirement is for a large between-groups effect size between the putative taxon and non-taxon members of Cohen's $d>1.25$ [13, 47]. All of the items meet this assumption (Table 2). The third assumption is that there is little nuisance covariance, which refers to the correlations between indicator variables within the taxon and non-taxon groups. A correlation of $r<.3$ has previously been recommended, and that the correlation between items across the whole sample is greater than the correlation between items in the taxon [13]. Neither of the measures met this assumption so we constructed composite indicator variables by summing scores across groups of items. Generalized least squares factor analyses were carried out on the scores of taxon members to determine which items should be combined. The factor loadings and the composite indicator variables are included in Tables 3 and 4. The factor loadings for the PGSI are straightforward (Table 3). The loadings from the adapted DSM measure revealed a three factor solution (Table 4). Two items loaded onto a first factor, and a further three items cross loaded onto this factor and a second factor. Three items loaded onto a third factor. Two other items did not load onto any of the factors and were included as indicators separately. All of these items have a sufficiently large between-groups effect size to be appropriate to detect the presence of a taxon (Tables 5-6). With the exception of two pairs of indicators all of the items met this assumption (Table 6).

PGSI Analysis

Both the MAMBAC and MAXSLOPE analyses indicated support for a categorical structure. Although neither set of comparison data was a close fit to the observed data in the MAXSLOPE analysis (Figure 1), the observed data are within the range of the categorical bootstrapped data. A CCFI $=0.633$ indicated support for a categorical structure. The 
MAMBAC analysis (Figure 1) shows stronger support in the same direction; the bootstrapped dimensional data is a poor fit of the averaged MAMBAC curve from the observed data, and a CCFI of .756 indicated strong support for a categorical interpretation.

Adapted DSM-IV Measure Analysis

The mean MAMBAC, MAXEIG and MAXCOV curves show a distinct peak on the right side of the x-axis that is characteristic of a taxon. Closer consideration of the bootstrapped data reveals that both categorical and dimensional datasets produce similar curves. Consequently we used CCFI's to discriminate between these interpretations. The MAMBAC analysis (Figure 2) strongly supports a categorical structure with the exception that analyses conducted on the first indicator were inconsistent. The computed CCFI across all curves was 0.717 , indicating support for a categorical interpretation. The MAXCOV and MAXEIG comparison curves (Figures 2) demonstrate that the categorical comparison data is closely calibrated with the observed data. The CCFI's also support this observation, with both $(\mathrm{MAXEIG}=0.756, \mathrm{MAXCOV}=0.811)$ indicating strong support for a categorical interpretation.

However, these analyses preclude us from making strong claims about the presence of a problem gambling taxon. To follow up this, we conducted a MAMBAC and MAXSLOPE analysis on the total scores of the PGSI and adapted DSM-IV criteria $(N=$ 1,486). We assigned cases to the putative taxon if they were classified as a problem gambler by either measure $(N=78)$. We note however that the two measures are highly correlated $(r=$ 0.736 (whole sample), 0.453 (taxon), 0.38 (non-taxon)), and so it is recommended this analysis is taken with extreme caution. Meehl has previously noted that high nuisance $(r>3)$ covariance should be tolerable so long as correlations are similar across both groups [48]. 
The analysis reveals very similar results to the analyses of the individual measures; the data provide stronger support for a categorical interpretation (MAMBAC CCFI $=0.628$, MAXSLOPE CCFI $=0.567$, Figure $3^{1}$ ).

\section{Discussion}

The taxometric analysis of the PGSI and a measured derived from the DSM-IV Pathological Gambling criteria from the BGPS 2010 indicated that problem gambling as measured by these instruments is categorical. Specifically, the PGSI analysis located a division that calibrates well to the cut-off for problem gambler in the PGSI (8+) and supports the assumptions underlying the Pathways Model, particularly the claim that there is a distinct group of problem gamblers characterised by a loss of control over their gambling $[5,6]$. Follow up analyses comparing across both scales supported this, further finding support for the presence of a categorical latent structure.

The PGSI was developed and is used on the assumption that it is measuring a dimension of harm-centred problem gambling. Our analysis shows that this assumption is flawed. The PGSI data from the BGPS demonstrates that the construct the PGSI is measuring is categorical, resembling a pathological model. Criticisms have previously been raised that the use of the PGSI is flawed because it is atheoretical [1]. Although such a claim is beyond the scope of this analysis, the PGSI does appear to measure a construct that probes aspects of the pathological model $[5]^{2}$. Given the poor performance of DSM-IV derived items related to losing control, it seems to be the case that the PGSI is a more conceptually coherent measure of the construct that the DSM-IV is intended to measure.

An indicator composed of items measuring a loss of control was also created for the adapted DSM-IV criteria (Indicator 1). In contrast to the PGSI, the results from this indicator variable were inconsistent. The observed difference between the PGSI and DSM 
constructs relating to a loss of control appears to be because the first two items on the DSMIV measure are frequently endorsed and do not discriminate between problem and nonproblem gamblers $[10,11]$.

The close similarity between the comparison curves on the analyses of the DSMIV based measure should be noted. Although the CCFIs are suitably large to endorse a categorical interpretation, indices obtained from the analysis belie the similarity between the two sets of bootstrapped data (Figures 2). Although the DSM indicators are skewed, comparison data have proved highly capable of discriminating between dimensional and categorical structures in data that are substantially skewed [40]; the comparison data here strongly support a categorical conclusion.

It would be beneficial to analyse other DSM datasets, however few are suitable because the DSM is usually measured dichotomously and may be unsuitable for taxometric analyses. Further analyses also do not resolve the underlying problem that response rates for at least two DSM items are relatively high in both problem and non-problem gamblers alike. Moreover, the response rates for three additional items that form the fifth DSM indicator (see Table 2) are higher in severe problem gamblers and are highly positively skewed. However, a limitation of this analysis is that sampling the general population means the base rate of problem gambling is low and cross-validation with a clinical sample may be beneficial. Also, it appears that self-report measures of the DSM (such as the one used here) may have a different factor structure to interview/clinician-based assessments [37]. Taxometric analyses are optimal in samples where the proportion of category to non category members is 50:50, although meaningful taxa can be identified with a base rate of 5\% (as in this analysis) [49].

In relation to other addiction disorders, the analysis provides further justification for the re-categorization of problem gambling as an addiction disorder in the DSM-V. Not only does problem gambling share strong similarities to substance use disorders [22], 
problem gamblers appear to form a taxon like other addictions. One implication is the need to carry out further psychometric assessments of both the PGSI and DSM-derived measures of problem gambling. Previous analyses used methods that are based on the assumption that the latent variable that is being measured is dimensional $[10,11]$. Our taxometric analysis of the BGPS demonstrates that this assumption is flawed. As such, the key implication is that different psychometric analyses, with different assumptions about latent models (such as latent profile analysis) are more appropriate for the psychometric evaluation of these measures.

\section{Conclusions}

A taxometric analysis was carried out on two problem gambling screens from the BGPS 2010, the PGSI and items from the DSM-IV Pathological Gambling criteria, as there was strong evidence in both theoretical and empirical research in problem gambling to hypothesize the presence of a taxon. The taxometric analyses demonstrated that the construct both scales probe is categorical in nature. The findings of this analysis have implications for the future measurement of problem gambling, and the psychometric methods used on these assessments. The findings also have implications for the classification of problem gambling as a behavioural addiction, demonstrating further empirical evidence that problem gambling shares a similar latent structure to other addiction disorders. 


\section{References}

1. Svetieva E, Walker M. Inconsistency between concept and measurement: The Canadian Problem Gambling Index (CPGI). Journal of Gambling Issues 2008;22:157-173.

2. Wardle H, Moody A, Spence S, Orford J, Volberg R, Jotangia D, et al. British Gambling Prevalence Survey 2010. Report prepared for the Gambling Commission; 2011.

3. Ferris J, Wynne H. The Canadian Problem Gambling Index: Final Report. Ottawa, ON: Canadian Centre on Substance Abuse; 2001.

4. American Psychiatric Association. Diagnostic and Statistical Manual of Mental Disorders (4th Ed., Text Revision). Washington, DC: American Psychiatric Association; 2000.

5. Rosecrance J. "The next best thing": a study of problem gambling. International Journal of the Addictions 1985;20:1727-1739.

6. Blaszczynski A, Nower L. A pathways model of problem and pathological gambling. Addiction 2002;97:487-499.

7. Milosevic A, Ledgerwood DM. The subtyping of pathological gambling: a comprehensive review. Clinical Psychology Review 2010;30:988-998.

8. Nower L, Martins SS, Lin K-H, Blanco C. Subtypes of Disordered Gamblers: Results from the National Epidemiologic Survey on Alcohol and Related Conditions. Addiction 2012;108:789-798.

9. Neal P, Delfabbro P, O’Neil M. Problem Gambling and Harm: Towards a National Definition. Melbourne: Gambling Research Australia; 2005.

10. Strong DR, Kahler CW. Evaluation of the continuum of gambling problems using the DSM-IV. Addiction 2007;102:713-721.

11. Orford J, Wardle H, Griffiths M, Sproston K, Erens B. PGSI and DSM-IV in the 2007 British Gambling Prevalence Survey: reliability, item response, factor structure and interscale agreement. International Gambling Studies 2010;10:31-44. 
12. Williams RJ, Volberg RA, Stevens RMG. The Population Prevalence of Problem Gambling: Methodological Influences, Standardized Rates, Jurisdictional Differences, and Worldwide Trends. Report Prepared for the Ontario Problem Gambling Research Centre and the Ontario Ministry of Health and Long Term Care; 2012.

13. Ruscio J, Haslam N, Ruscio AM. Introduction to the Taxometric Method: A Practical Guide. Hove, UK: Routledge; 2006.

14. Gambino B. Setting Criterion Thresholds for Estimating Prevalence: What is Being Validated? Journal of Gambling Studies 2013. DOI 10.1007/s10899-013-9380-y

15. Gambino B. The Validation of Screening Tests: Meet the New Screen same as the Old Screen? Journal of Gambling Studies 2011;28:573-605.

16. Walker M, Blaszczynski A. Clinical Assessment of Problem Gamblers Identified Using the Canadian Problem Gambling Index. Adelaide: Independent Gambling Authority; 2010 .

17. Currie SR, Casey DM, Hodgins DC. Improving the Psychometric Properties of the Problem Gambling Severity Index. Report prepared for the Canadian Consortium for Gambling Research; 2010.

18. Currie SR, Hodgins DC, Casey DM. Validity of the Problem Gambling Severity Index interpretive categories. Journal of Gambling Studies 2013;29:311-327.

19. Kincaid H, Daniels R, Dellis A, Hofmeyr A, Rousseau J, Sharp C, et al. A Taxometric Analysis of Problem Gambling Data from a South African National Urban Sample. Journal of Gambling Studies 2012. DOI 10.1007/s10899-012-9316-y

20. Lesieur HR, Blume SB. The South Oaks Gambling Screen (SOGS): A new instrument for the identification of pathological gamblers. American Journal of Psychiatry 1987;144:1184-1188.

21. Petry NM, Blanco C, Auriacombe M, Borges G, Bucholz K, Crowley TJ, et al. An Overview of and Rationale for Changes Proposed for Pathological Gambling in DSM-5. Journal of Gambling Studies 2013. 
22. Denis C, Fatséas M, Auriacombe M. Analyses related to the development of DSM-5 criteria for substance use related disorders: 3. An assessment of Pathological Gambling criteria. Drug and Alcohol Dependence 2012;122:22-27.

23. Haslam N, Holland E, Kuppens P. Categories versus dimensions in personality and psychopathology: a quantitative review of taxometric research. Psychological Medicine 2012;42:903-920.

24. Baillie AJ, Teesson M. Continuous, categorical and mixture models of DSM-IV alcohol and cannabis use disorders in the Australian community. Addiction 2010:105;12461253

25. Slade T, Grove R, Teesson M. A taxometric study of alcohol abuse and dependence in a general population sample: evidence of dimensional latent structure and implications for DSM-V. Addiction 2009;104:742-751.

26. Ginestet CE, Mitchell K. Wellman N. Taxometric investigation of the latent structure of nicotine dependence: An epidemiological sample. Nicotine \& Tobacco Research 2008; $10: 833-841$.

27. Denson TF, Earleywine M. Pothead or pot smoker? A taxometric investigation of cannabis dependence. Substance Abuse Treatment, Prevention and Policy 2006: 1; 22.

28. McBride O, Adamson G, Shevlin M. A latent class analysis of DSM-IV pathological gambling criteria in a nationally representative British Sample. Psychiatry Research 2010: $178 ; 401-407$.

29. Braverman J, LaBrie RA, Shaffer HJ. A taxometric analysis of actual Internet sports gambling behavior. Psychological Assessment 2011;23:234-244.

30. Ross D, Dellis A, Hofmeyr A, Kincaid H, Sharp C, Vucinich R. South African National Urban Prevalence Study of Gambling Behavior.

http://www.academia.edu/1191790/National_Urban_Prevalence_Study_of_Gambling_Behav iour_in_South_Africa_NUPSGB_Summary_Data (accessed 20 August 2013) (Archived at http://www.webcitation.org/6J2EazBT1 on 21 August 2013). 
31. Walters GD, Ruscio J. To sum or not to sum: taxometric analysis with ordered categorical assessment items. Psychological Assessment 2009;21:99-111.

32. UK Data Service. British Gambling Prevalence Survey 2010.

http://discover.ukdataservice.ac.uk/catalogue?sn=6843. (accessed 14 August 2013) (Archived at http://www.webcitation.org/6J2EVmoMd on 21 August 2013).

33. Williams RJ, Volberg RA. Best practices in the population assessment of problem gambling. Report prepared for the Ontario Problem Gambling Research Center; 2010.

34. Sproston K, Erens B, Orford J. Gambling Behaviour in Britain: Results from the British Gambling Prevalence Survey. Report prepared for the Gambling Commission; 1999.

35. Wardle H, Sproston K, Orford J, Erens B, Griffiths M, Constantine R, Pigott S. British Gambling Prevalence Survey 2007. Report prepared for the Gambling Commission; 2007.

36. Fisher S. Gambling and problem gambling among casino patrons. Report prepared for the British Casino Industry Consortium; 1996.

37. Stinchfield R, Govoni R, Frish G. DSM-IV Diagnostic Criteria for Pathological Gambling: Reliability, Validity and Classification Accuracy. The American Journal of Addictions 2005; 14:73-82

38. Stinchfield R. Reliability, Validity and Classification Accuracy of a Measure of DSM-IV Diagnostic Criteria for Pathological Gambling. American Journal of Psychiatry 2003; 160: 180-182.

39. Orford J, Sproston K, Erens B. SOGS and DSM-IV in the British gambling prevalence survey: Reliability and factor structure. International Gambling Studies; 3: 53-65.

40. Meehl PE, Yonce LJ. Taxometric analysis: I. Detecting taxonicity with two quantitative indicators using means above and below a sliding cut (MAMBAC procedure). Psychological Reports 1994;74:1059-1274.

41. Grove WM. The MAXSLOPE Taxometric Procedure: Mathematical Derivation, Parameter Estimation, Consistency Tests. Psychological Reports 2004;95:517-50. 
42. Meehl PE. MAXCOV-HITMAX: A Taxonomic Search Method for Loose Genetic Syndromes. Psychodiagnosis: Selected Papers, Minneapolis: University of Minnesota Press; 1973, p. 200-224.

43. Waller NG, Meehl PE. Multivariate Taxometric Procedures: Distinguishing Types from Continua. Thousand Oaks, CA: Sage Publications; 1998.

44. Walters GD, Ruscio J. Where do we draw the line? Assigning cases to subsamples for MAMBAC, MAXCOV, and MAXEIG taxometric analyses. Assessment 2010;17:321-333.

45. Ruscio J, Ruscio AM, Keane TM. Using taxometric analysis to distinguish a small latent taxon from a latent dimension with positively skewed indicators: the case of involuntary defeat syndrome. Journal of Abnormal Psychology 2004;113:145-154.

46. Ruscio J. Taxometrics and related programs. http://www.tcnj.edu/ ruscio/taxometrics.html (accessed 14 August 2013) (Archived at http://www.webcitation.org/6J2EMyXSO on 21 August 2013).

47. Meehl PE. Clarifications about taxometric method. Applied and Preventive Psychology: 1999;8:165-174.

48. Meehl PE. Bootstraps taxometrics. Solving the classification problem in psychopathology. American Psychologist 1995; 50: 266-275

49. Ruscio J, Marcus DK. Detecting small taxa using simulated comparison data: A reanalysis of Beach, Amir and Bau's (2005) data. Psychological Assessment 2007; 19: 241246. 


\section{Footnotes}

1. In figure 3 we have included the MAXSLOPE curve as well as the comparison curve because the comparison curve is very difficult to interpret because the range of the dimensional data is so high.

2. In addition to the claims that problem gambling is a categorical disorder and is demarcated by a loss of control of gambling behaviour, there are three other claims [5]. The remaining claims are that problem gambling is a single phenomenon, compulsive gambling is a permanent and irreversible disorder, and that the disorder progresses through a series of stages. The progression of gambling behaviour begins with a transition from recreational to excessive gambling, followed by the appearance of cognitive biases and overconfidence. This in turn progresses changes in perceptions of value of money, then chasing losses, followed by the consequences of problem gambling, then a 'rock bottom' stage when treatment is sought. Although two of these claims, that problem gambling is a single phenomenon and irreversible are not empirically supported, the remaining claim is tested by the DSM-IV measure. 
A

Categorical Comparison Data

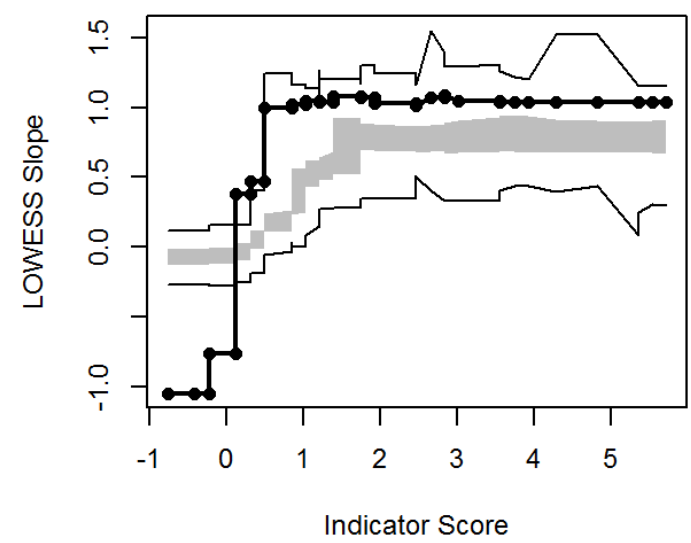

B

Categorical Comparison Data

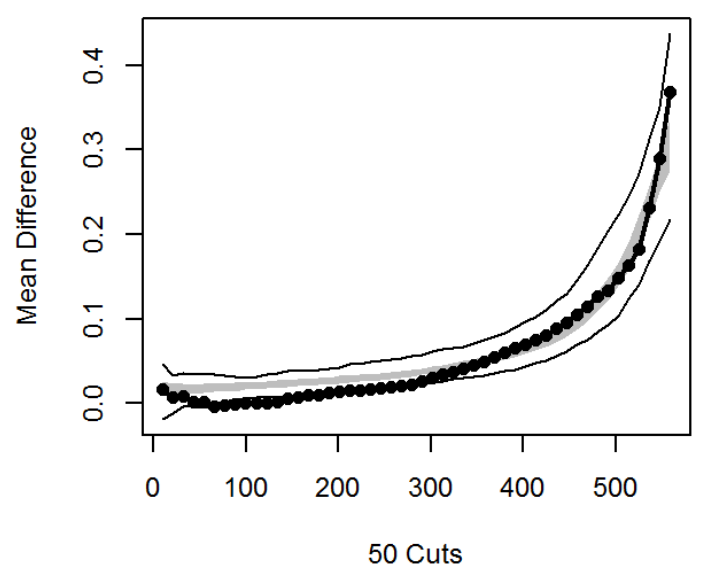

Dimensional Comparison Data

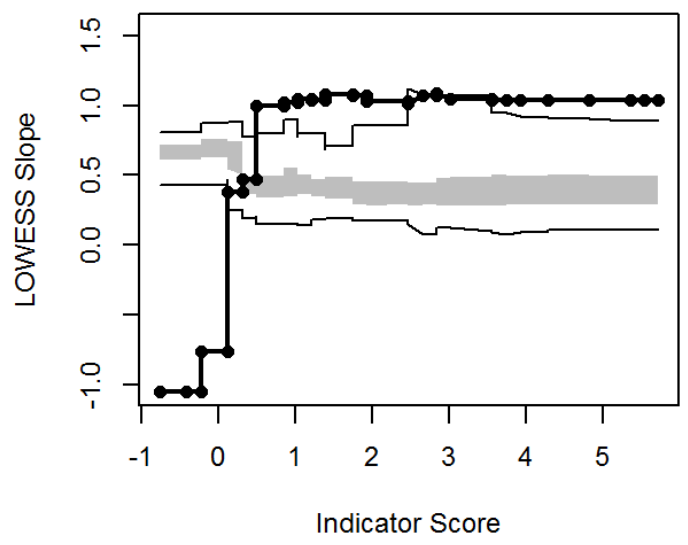

Dimensional Comparison Data

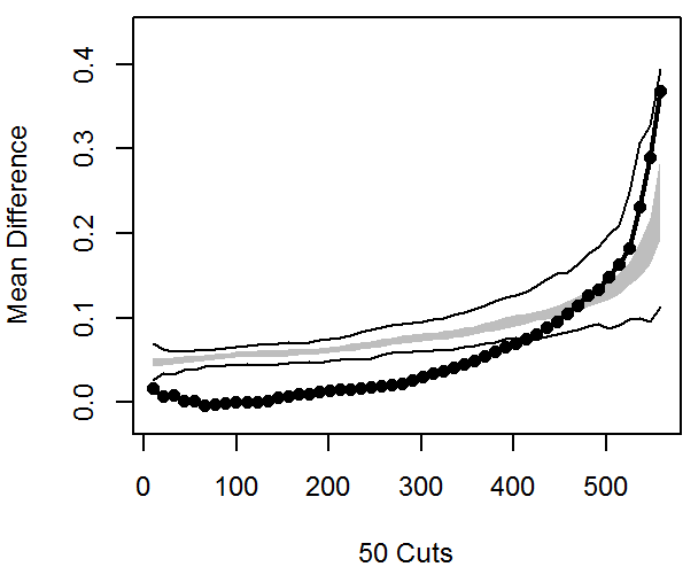


A
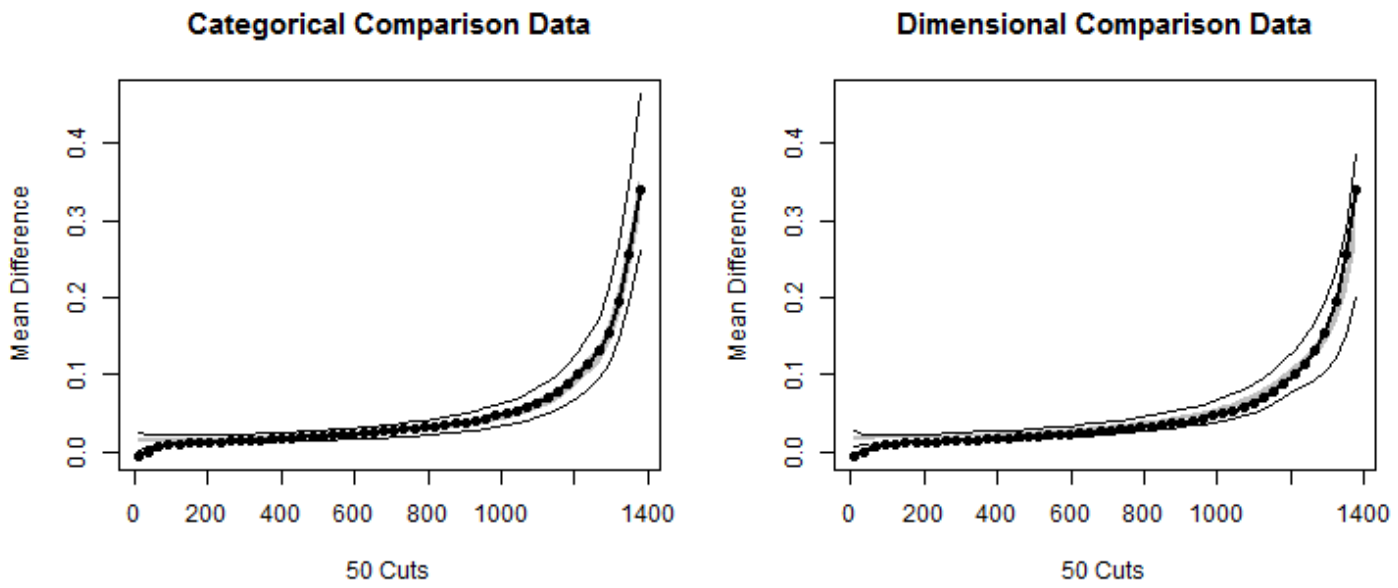

B

Categorical Comparison Data

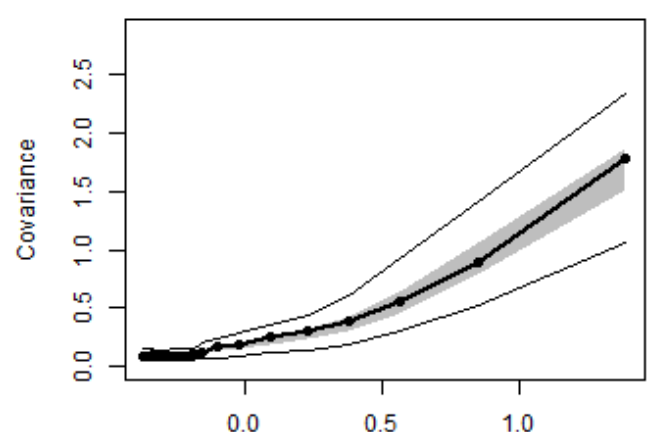

50 Windows

C

Categorical Comparison Data

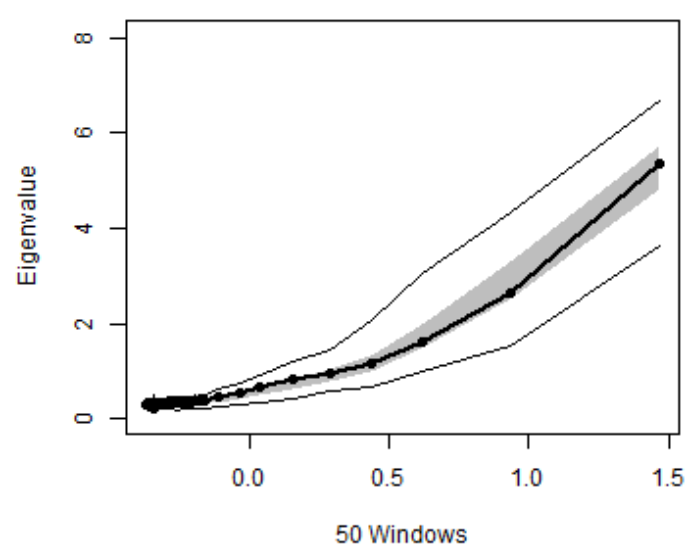

Dimensional Comparison Data

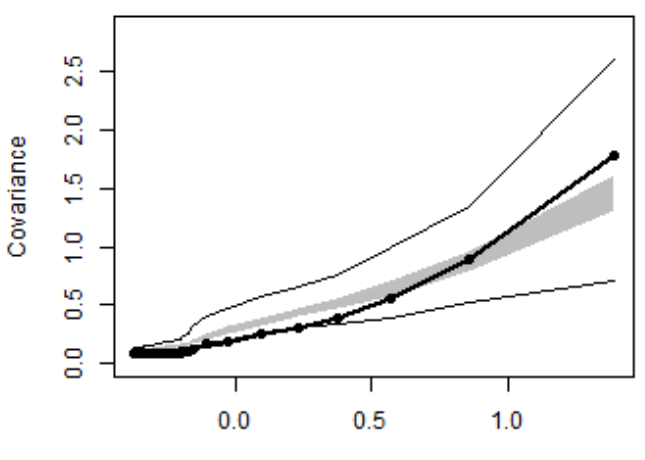

50 Windows

Dimensional Comparison Data

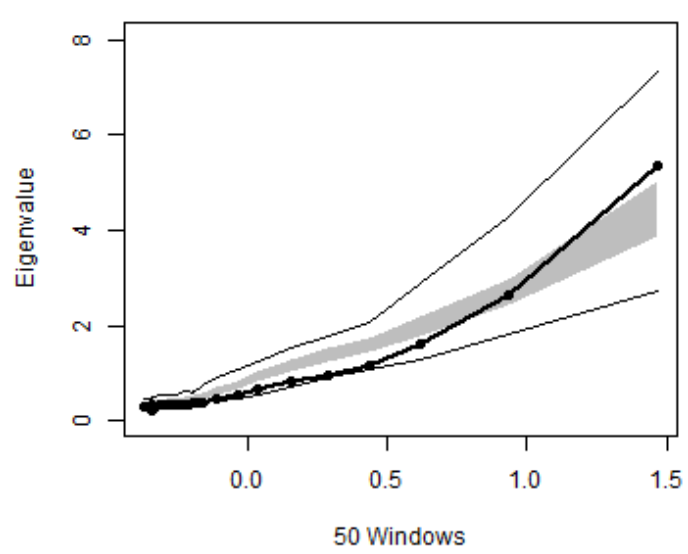



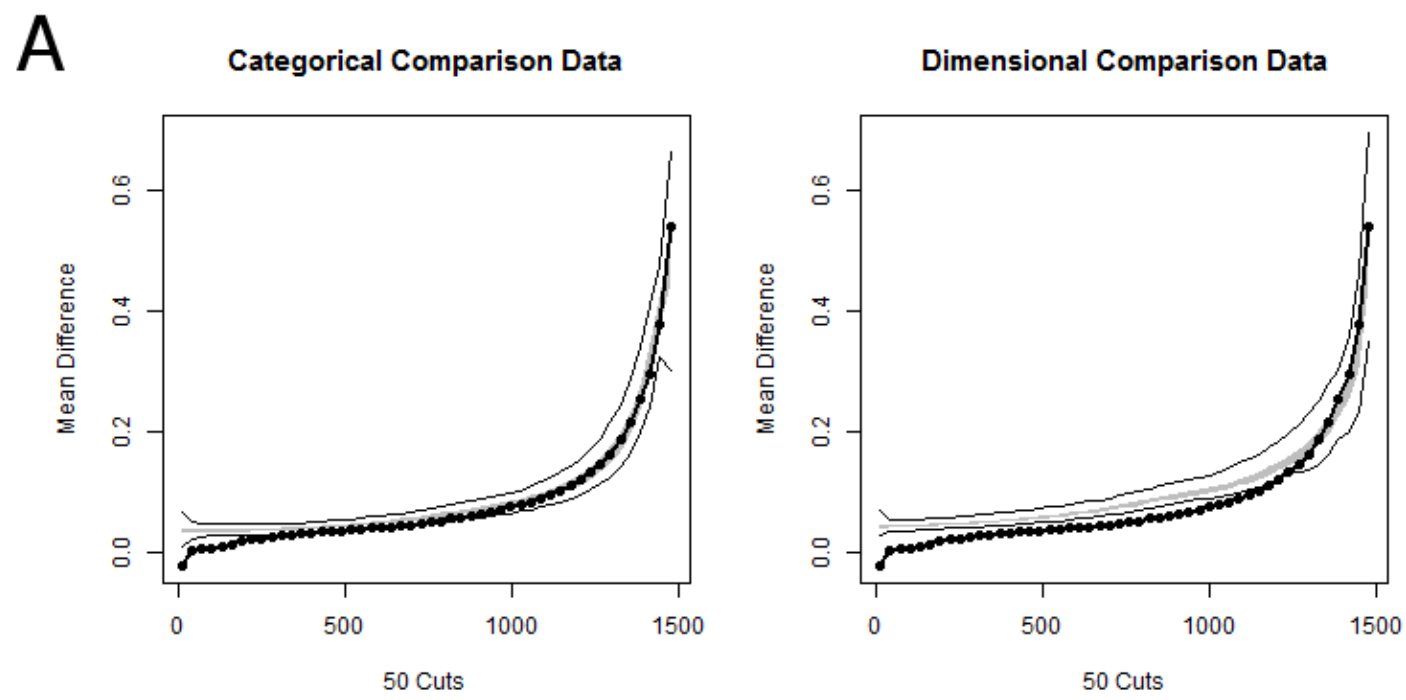

B
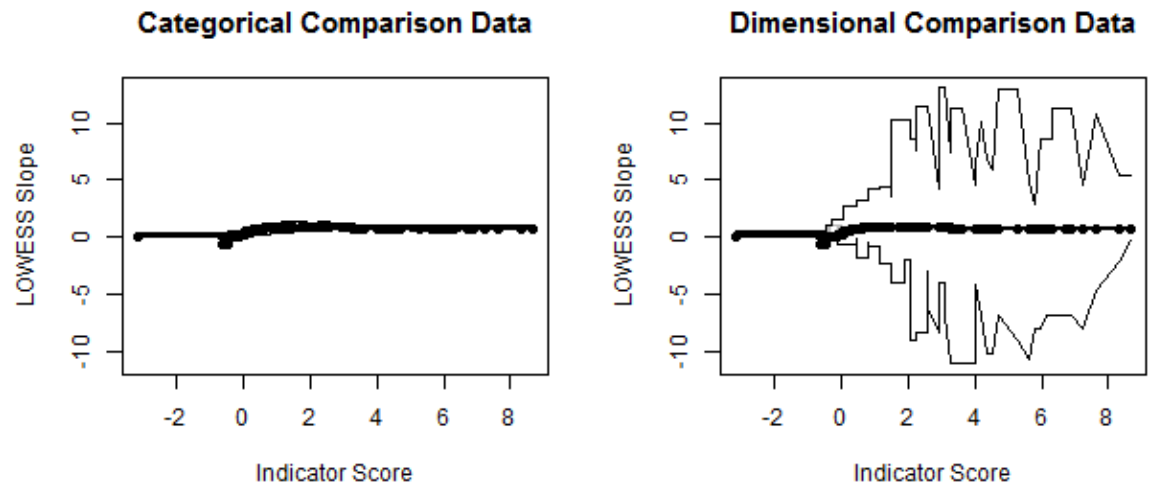

C

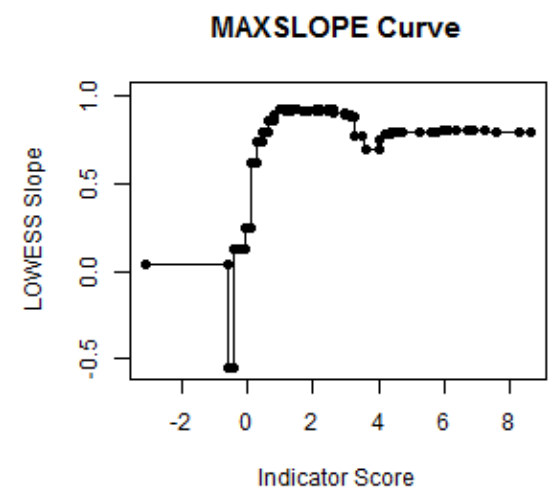




\section{Figure Legends}

Figure 1. Categorical and dimensional comparison data compared against the observed data points for the PGSI MAXSLOPE (Graph A, CCFI = 0.633) and MAMBAC (Graph B, CCFI $=0.756)$ analyses. The grey band represents the middle $50 \%$ of the data points from 100 bootstrapped samples $(\mathrm{N}=100,000)$ with categorical and dimensional properties, with the same statistical distributions as the observed data. The two black lines represent the maximum and minimum points from the bootstrapped sample. The dotted black line is the averaged MAXSLOPE curve from the actual data observed.

Figure 2. Categorical and dimensional comparison data compared against the observed data points for the DSM-IV MAMBAC (Graph A, CCFI =0.717), MAXCOV $($ Graph B, CCFI $=0.811)$ and MAXEIG (Graph C, CCFI $=0.756)$ analyses. The grey band represents the middle $50 \%$ of the data points from 100 bootstrapped samples $(\mathrm{N}=100,000)$ with categorical and dimensional properties, with the same statistical distributions as the observed data. The two black lines represent the maximum and minimum points from the bootstrapped sample. The dotted black line is the averaged MAMBAC curve from the actual data observed.

Figure 3. Categorical and dimensional comparison data compared against the observed data points for the DSM-IV and PGSI MAMBAC (Graph A, CCFI $=0.628$ ) and MAXSLOPE (Graph B, CCFI = 0.567). The grey band represents the middle $50 \%$ of the data points from 100 bootstrapped samples $(\mathrm{N}=100,000)$ with categorical and dimensional properties, with the same statistical distributions as the observed data. The two black lines represent the maximum and minimum points from the bootstrapped sample. The dotted black line is the averaged MAMBAC curve from the actual data observed. The MAXSLOPE curve (Graph C) without comparison data because of the range of dimensional comparison data observed. 
Table 1. Means, standard deviations and ranges for the items on both screens.

\begin{tabular}{|c|c|c|}
\hline Item & Average & S.D. \\
\hline \multicolumn{3}{|c|}{ Problem Gambling Severity Index } \\
\hline 1 & 0.53 & 0.719 \\
\hline 2 & 0.21 & 0.514 \\
\hline 3 & 0.67 & 0.684 \\
\hline 4 & 0.15 & 0.476 \\
\hline 5 & 0.24 & 0.589 \\
\hline 6 & 0.19 & 0.529 \\
\hline 7 & 0.32 & 0.628 \\
\hline 8 & 0.19 & 0.559 \\
\hline 9 & 0.39 & 0.673 \\
\hline \multicolumn{3}{|c|}{$\begin{array}{l}\text { Adapted DSM-IV Pathological } \\
\text { Gambling Criteria }\end{array}$} \\
\hline 1 & 0.56 & .788 \\
\hline 2 & 0.94 & .665 \\
\hline 3 & 0.27 & .586 \\
\hline 4 & 0.15 & .508 \\
\hline 5 & 0.19 & .525 \\
\hline 6 & 0.12 & .433 \\
\hline 7 & 0.15 & .514 \\
\hline 8 & 0.02 & .285 \\
\hline 9 & 0.03 & .253 \\
\hline 10 & 0.05 & .281 \\
\hline
\end{tabular}

Note: The ranges for all of the items on both measures are $0-3$. 
Table 2. Indicator validity and skew measures for individual items on the PGSI and adapted DSM-IV criteria

\section{Problem Gambling Severity Index}

1. How often have you bet more than you can afford to lose?

2. How often have you needed to gamble with larger amounts of money

to get the same feeling of excitement?

3. How often have you gone back another day to try to win back the money you lost?

4. How often have you borrowed money or sold anything to get money to gamble?

5 . How often have you felt you might have a problem with gambling?

6 . How often have people criticized your betting or told you that you had a gambling problem, regardless of whether or not you thought it was true?

7. How often have you felt guilty about gambling, or what happens when you gamble?

8. How often has your gambling caused you any health problems, including stress or anxiety?

9. How often has gambling caused any financial problems for you or your household?

\section{Adapted DSM-IV Pathological Gambling Criteria}

1. In the last 12 months, how often do you go back another day to win back money you lost?

2. In the last 12 months, how often have you found yourself thinking about gambling (that is reliving past gambling experiences, planning the next time you will play or thinking of ways to get money to gamble)? 3 . In the last 12 months, have you needed to gamble with more and

7. In the last 12 months, have made unsuccessful attempts to control, cut back or stop gambling?

8. In the last 12 months, have you committed a crime in order to finance gambling or pay gambling debts?

9. In the last 12 months, have you risked or lost an important 
Table 3. Factor loadings and indicator names from generalized least squares factor analysis on PGSI taxon members.

\begin{tabular}{|c|c|c|}
\hline PGSI Item Number and Content & Factor 1 & Factor 2 \\
\hline $\begin{array}{l}\text { Indicator } 1 \\
1 . \text { How often have you bet more than you could } \\
\text { afford to lose? }\end{array}$ & .274 & .801 \\
\hline $\begin{array}{l}\text { 2. How often have you needed to gamble with } \\
\text { larger amounts of money to get the same feeling of } \\
\text { excitement? }\end{array}$ & .170 & .602 \\
\hline $\begin{array}{l}\text { 3. How often have you gone back another day } \\
\text { to try to win back the money you lost? } \\
\text { Indicator } 2\end{array}$ & .305 & .595 \\
\hline $\begin{array}{l}\text { 4. How often have you borrowed money or sold } \\
\text { anything to get money to gamble? }\end{array}$ & .545 & .100 \\
\hline $\begin{array}{l}\text { 5. How often have you felt that you might have } \\
\text { a problem with gambling? }\end{array}$ & .719 & .140 \\
\hline $\begin{array}{l}\text { 6. How often have people criticized your } \\
\text { betting or told you that you had a gambling problem, } \\
\text { regardless of whether or not you thought it was true? }\end{array}$ & .726 & -.011 \\
\hline $\begin{array}{l}\text { 7. How often have you felt guilty about } \\
\text { gambling or what happens when you gamble? }\end{array}$ & .594 & -.279 \\
\hline $\begin{array}{l}\text { 8. How often has your gambling caused you } \\
\text { any health problems, including stress or anxiety? }\end{array}$ & .950 & -.147 \\
\hline $\begin{array}{l}\text { 9. How often has gambling caused any financial } \\
\text { problems for you or your household? }\end{array}$ & 682 & .210 \\
\hline
\end{tabular}


Table 4. Factor loadings and indicator names from generalized least squares factor analysis on DSM-IV taxon members.

\begin{tabular}{|c|c|c|c|c|}
\hline & DSM-IV Item Number and Content & Factor 1 & Factor 2 & Factor 3 \\
\hline \multicolumn{2}{|c|}{ Indicator 1} & .202 & -.083 & .449 \\
\hline 1. & $\begin{array}{l}\text { In the last } 12 \text { months, how often do you go back } \\
\text { another day to win back money you lost? }\end{array}$ & & & \\
\hline 2. & $\begin{array}{l}\text { In the last } 12 \text { months, how often have you found } \\
\text { yourself thinking about gambling (that is } \\
\text { reliving past gambling experiences, planning the } \\
\text { next time you will play, or thinking of ways to } \\
\text { get money to gamble)? }\end{array}$ & .005 & .064 & .657 \\
\hline 3. & $\begin{array}{l}\text { In the last } 12 \text { months, have you needed to } \\
\text { gamble with more and more money to get the } \\
\text { excitement you are looking for? }\end{array}$ & .237 & .266 & .534 \\
\hline \multicolumn{5}{|c|}{ Indicator 2} \\
\hline 4. & $\begin{array}{l}\text { In the last } 12 \text { months, have you felt restless or } \\
\text { irritable when trying to cut down gambling? }\end{array}$ & .318 & .231 & .385 \\
\hline \multicolumn{5}{|c|}{ Indicator 3} \\
\hline 5. & $\begin{array}{l}\text { In the last } 12 \text { months, have you gambled to } \\
\text { escape from problems or when you are feeling } \\
\text { depressed, anxious or bad about yourself? }\end{array}$ & .263 & .107 & .231 \\
\hline \multicolumn{5}{|c|}{ Indicator 4} \\
\hline 6. & $\begin{array}{l}\text { In the last } 12 \text { months, have you lied to family, to } \\
\text { others, to hide the extent of your gambling? }\end{array}$ & .941 & -.222 & -.039 \\
\hline 7. & $\begin{array}{l}\text { In the last } 12 \text { months, have you made } \\
\text { unsuccessful attempts to control, cut back or } \\
\text { stop gambling? }\end{array}$ & .516 & -.065 & .280 \\
\hline \multicolumn{5}{|c|}{ Indicator 5} \\
\hline 8. & $\begin{array}{l}\text { In the last } 12 \text { months, have you committed a } \\
\text { crime in order to finance gambling or pay } \\
\text { gambling debts? }\end{array}$ & .356 & 648 & -.165 \\
\hline 9. & $\begin{array}{l}\text { In the last } 12 \text { months, have you risked or lost an } \\
\text { important relationship, job, educational or work } \\
\text { opportunity because of gambling? }\end{array}$ & .434 & .788 & -.049 \\
\hline 10. & $\begin{array}{l}\text { In the last } 12 \text { months, have you asked others to } \\
\text { provide money to help with a desperate } \\
\text { financial situation cause by gambling? }\end{array}$ & .552 & .445 & -.008 \\
\hline
\end{tabular}


Table 5. Indicator validity and skew measures for composite indicator variables for the PGSI and adapted DSM-IV criteria, and the nuisance covariance for the PGSI measure.

\begin{tabular}{|c|c|c|c|}
\hline Indicator & Cohen's $d$ & & Skew \\
\hline \multicolumn{4}{|l|}{ Problem Gambling Severity Index } \\
\hline 1 (PGSI Items 1-3) & 3.008 & & 2.424 \\
\hline 2 (PGSI Items 4-9) & 4.388 & & 3.411 \\
\hline \multicolumn{4}{|l|}{ Adapted DSM-IV Pathological } \\
\hline \multicolumn{4}{|l|}{ Gambling Criteria } \\
\hline 1 (DSM Items 1-3) & 3.635 & & 2.125 \\
\hline $2(\mathrm{DSM}$ Item 4) & 3.806 & & 3.830 \\
\hline 3 (DSM Item 5) & 3.791 & & 3.385 \\
\hline 4 (DSM Items 6-7) & 3.868 & & 3.993 \\
\hline \multirow[t]{2}{*}{5 (DSM Items 8-10) } & 2.985 & & 8.930 \\
\hline & \multicolumn{3}{|c|}{$r$} \\
\hline PGSI Indicators $1 \& 2$ - Whole Sample & \multicolumn{3}{|c|}{0.555} \\
\hline \multicolumn{4}{|l|}{ Covariance } \\
\hline PGSI Indicators $1 \& 2$ - Taxon Nuisance & \multicolumn{3}{|c|}{0.288} \\
\hline \multicolumn{4}{|l|}{ Covariance } \\
\hline PGSI Indicators $1 \& 2$ - Non-taxon & \multicolumn{3}{|c|}{-0.054} \\
\hline Nuisance Covariance & & & \\
\hline
\end{tabular}


Table 6. Inter-item correlations for the DSM-IV

Whole sample

\begin{tabular}{ccccccc}
\hline & 1 & 2 & 3 & 4 & 5 \\
\hline 1 & - & & & & \\
2 & 0.517 & - & & & \\
3 & 0.437 & 0.505 & - & & & \\
4 & 0.484 & 0.560 & 0.517 & - & & \\
5 & 0.352 & 0.452 & 0.407 & 0.473 & - & \\
\hline Taxon & & & & & & \\
\hline & 1 & & & & \\
\hline 1 & & & & & & \\
2 & $\mathbf{0 . 3 7 5}$ & - & & & \\
3 & 0.227 & 0.192 & - & & \\
4 & 0.255 & 0.278 & 0.229 & - & \\
5 & 0.148 & 0.300 & 0.170 & $\mathbf{0 . 3 3 0}$ & - \\
\hline
\end{tabular}

Non-taxon members

\begin{tabular}{cccccc}
\hline & 1 & 2 & 3 & 4 & 5 \\
\hline 1 & - & & & & \\
2 & 0.189 & - & & & \\
3 & 0.063 & 0.190 & - & & \\
4 & 0.147 & 0.273 & 0.191 & - & \\
5 & -0.009 & 0.062 & 0.069 & 0.075 & -
\end{tabular}

Note. The correlations that exceed the recommended nuisance covariance threshold of $r<.3$ are highlighted in bold. 